\title{
Partizipation als organisationales und pädagogisches Konzept der Elterninitiativen - oder: ,[...] das war eher so ein exklusiv-inklusiver Bereich"
}

\author{
Katharina Riechers
}

Eingegangen: 28. Oktober 2020 / Angenommen: 12. März 2021 / Online publiziert: 6. Juli 2021 (C) Der/die Autor(en) 2021

Zusammenfassung Der Beitrag fokussiert Elterninitiativen als besondere Form einer partizipativ durch Eltern und pädagogische Fachkräfte gestalteten Trägerschaft. Er fragt nach dem Zusammenhang ihrer organisationalen Strukturen und ihres pädagogisch-konzeptionellen Partizipationsverständnisses sowie nach Inklusionspotenzialen und Exklusionsrisiken, die sich über ihre spezielle Organisationsform ergeben (können), und verbindet diese mit verschiedenen inklusionstheoretischen Konzepten.

Schlüsselwörter Frühe Bildung, Betreuung und Erziehung (FBBE) ·

Elterninitiativ-Kindertageseinrichtungen · Elterliche Selbstorganisation · Kindliche Partizipation · Spannungsfeld In- und Exklusion

\section{Participation as the organizational and pedagogical concept of parent initiatives-or: "[...] that was more of an exclusively inclusive area"}

\begin{abstract}
The article focuses on parent initiatives as a hybrid form of private and public sponsorship, which is organised by parents and pedagogical staff. Based on current data from parent initiatives, the contribution analyses the interdependencies between their organizational structures and their pedagogical concept of participation and discusses the tension between processes of in- and exclusion, which can be resulting from the above.
\end{abstract}

Keywords Early childhood education and care · Parent initiatives · Parental selforganization · Children as participants · Tension between in- and exclusion

Katharina Riechers $(\bowtie)$

FB 1 Erziehungs- und Sozialwissenschaften, Institut für Erziehungswissenschaft/Abt. Allgemeine

Erziehungswissenschaft, Stiftung Universität Hildesheim, Universitätsplatz 1, 31141 Hildesheim,

Deutschland

E-Mail: riech002@uni-hildesheim.de

Beyersche Burg 18, 31137 Hildesheim, Deutschland 


\section{Einleitung}

Bei Elterninitiativen handelt es sich um Kindertageseinrichtungen, die engagierte Eltern als gemeinnützige Vereine gegründet haben und zusammen mit pädagogischen Fachkräften betreiben. Somit haben sowohl die Eltern als auch die pädagogischen Fachkräfte hier traditionell mehr Mitspracherecht, als dies bei anderen Trägerformen der Fall ist. Alle anfallenden Entscheidungen werden gemeinsam getroffen und arbeitsteilig, aber nicht-hierarchisch, umgesetzt. Damit bedient diese spezielle Form der Trägerschaft eine besondere Nische innerhalb der Bildungs- und Betreuungslandschaft. Als privat verantwortete Einrichtungen der Kindertagesbetreuung sind Elterninitiativen transorganisational zwischen Öffentlichkeit und Privatheit angesiedelt (Baader 2009; Baader und Bollig 2019) und können somit als ,,institutionalisierte Formen der geteilten Wohlfahrtsproduktion“ (ebd., S. 142) bezeichnet werden.

Doch nicht nur auf der organisatorischen, auch auf der pädagogischen Ebene kommt dem Aspekt der Teilhabe eine besondere Bedeutung zu: Kinder als Akteure $\mathrm{zu}$ verstehen und ihnen das aktive Mitgestalten aller sie betreffenden Lebensbereiche zuzugestehen, hat in Elterninitiativen seit jeher Tradition (Baader 2011; Baader und Sager 2010). Zudem gehörten sie zu den ersten Kindertageseinrichtungen, die bereits seit den 1980er-Jahren in Eigenengagement und zunächst ohne rechtliche Grundlage sogenannte ,Integrationsplätze‘ für Kinder mit Beeinträchtigungen geschaffen haben (Aden-Grossmann 2011; Albers et al. 2011; Baader 2014). Damit erweisen sie sich im Hinblick auf die Untersuchungsgegenstände Partizipation und Inklusion in vielfacher Hinsicht als interessante Institutionen.

Der vorliegende Beitrag umreißt zunächst knapp die entstehungsgeschichtlichen Zusammenhänge und pädagogisch-konzeptionellen Hintergründe, aus denen die heutigen Elterninitiativen hervorgegangen sind und zeigt anschließend ihre Weiterentwicklung, aktuelle Verbreitung, Vielfalt und gegenwärtigen organisationalen Strukturen auf (Kap. 2). Welche Zusammenhänge sich für die Elterninitiativen zwischen ihren partizipativ organisierten Strukturen und ihrer pädagogischen Konzeptualisierung von kindlicher Partizipation ergeben - und welche In- und Exklusionsprozesse aus ihren trägerspezifischen Strukturen resultieren (können) -, soll abschließend unter Bezugnahme auf das Projekt „Inklusive Elterninitiativen“1 (Kap. 3 und 4) diskutiert und abschließend kontrastierend gebündelt werden (Kap. 5).

\section{Von den Ursprüngen der Kinderläden zu den heutigen Elterninitiativen}

\subsection{Entstehungsgeschichtlicher Kontext}

Die historischen Wurzeln der Elterninitiativen sind in der Kinderladenbewegung der sogenannten '68er zu finden, bei der es sich um die erste Protestbewegung der bundesdeutschen Nachkriegsgeschichte handelte, die gezielt Erziehungsfragen

\footnotetext{
1 Leitung: Prof.'in Dr. Meike Sophia Baader; Laufzeit 2017-2021 (gefördert im Niedersächsischen Vorab vom Ministerium für Wissenschaft und Kultur und der Volkswagenstiftung).
} 
fokussierte (Baader 2007). Fragt man nach den Motiven der Protestbewegung, so kommen diese zum einen in der prekären Gesamtsituation der Kindertagesbetreuung der 1960er/70er-Jahre zum Ausdruck, die durch massiven Platzmangel und ein Defizit an pädagogischen Fachkräften gekennzeichnet war (Aden-Grossmann 2019; Baader 2008, 2020). Unter derartigen Bedingungen sei für die überforderten Fachkräfte lediglich ein autoritärer Erziehungsstil umsetzbar (Bott 1970), der wiederum für die „Entstehung einer zum Faschismus neigenden Persönlichkeit“ (Baader und Sager 2010, S. 258f.) verantwortlich gemacht wurde (Baader 2008, 2020). Damit kann die Gründung erster Kinderläden ${ }^{2}$ sowohl als eine vor allem von akademischen und linksorientierten Eltern initiierte Reaktion ,parentaler Selbstorganisation“ (Wolff 2019, S. 30) auf einen bildungs-/sozialpolitischen Missstand im Bereich der Vorschulerziehung als auch als ein Versuch der reflexiven Aufarbeitung der nationalsozialistischen Vergangenheit - über eine Abgrenzung von den Erziehungsmaximen der eigenen Elterngeneration - verstanden werden (Baader 2008, 2011, 2020; Jansa 2000).

Dabei oblag die Bereitstellung und Gestaltung der Räumlichkeiten sowie die Gewährleistung aller weiteren organisatorischen, praktischen und - zunächst auch pädagogischen Tätigkeiten allein der Verantwortlichkeit der Eltern (Baader und Bollig 2019; Berliner Kinderläden 1970; Bott 1970).

\subsection{Pädagogische Ansätze}

Auch wenn die pädagogischen Konzepte der Kinderläden, ihre theoretischen Bezüge und praktischen Umsetzungsformen variierten ${ }^{3}$, so ist es vor allem der Begriff der antiautoritären Erziehung, der mit ihnen verbunden wird und im öffentlichen Gedächtnis Fuß fassen konnte. Die antiautoritäre Erziehung versteht das Kind bereits ab dem Säuglingsalter als einen autonomen Menschen (Aden-Grossmann 2019); als „eine ,Person aus eigenen Rechten“ und mit eigenen Bedürfnissen“ (Baader und Sager 2010, S. 262), die es ausdrücken und verhandeln kann (Baader 2011). Der antiautoritären Erziehung geht es also zentral um die Selbstständigkeit, Selbstentfaltung und Selbstbestimmung des Kindes (Baader und Sager 2010; Aden-Grossmann 2019). Unter Bezugnahme auf Wilhelm Reich wird der Begriff der Selbstregulierung zum Schlagwort dessen, wofür die Erziehung der Kinderläden steht: Es galt ein Umfeld zu schaffen, in dem den Kindern Autonomie zugestanden und in weitgehend selbstgesteuerten individuellen und kollektiven Aushandlungs- und Lernprozessen bedürfnisorientiert verhandelt wird (ebd. sowie Baader 2011, 2020; Bock et al. 2019). In der konkreten pädagogischen Handlungspraxis bedeutete dies, dass die Erziehung und das Lernen vom Kind ausgehen sollten (Aden-Grossmann 2019; Bock et al. 2019; Seifert 1977). Damit wurden Kinder ,zu Akteuren ihrer eigenen

\footnotetext{
2 Ihren Namen verdanken die Kinderläden leerstehenden Ladengeschäften, in denen die ersten Initiativen in Berlin kostengünstig unterkamen (Berliner Kinderläden 1970); ein Modell, das sich auch in anderen Regionen durchsetzte und z. T. bis heute Bestand hat.

3 So verweist Baader (2009) auf eine Typisierung in sozialistisch, psychoanalytisch, pragmatisch-liberal und demokratisch konzipierte Kinderläden. Für vertiefende Darstellungen zu den unterschiedlichen Strömungen und deren theoretischen Bezugspunkten siehe ebd. (2020) sowie Baader und Sager (2010).
} 
Bedürfnisse erklärt“ und somit ,,aus pädagogischer Perspektive als Akteure konstituiert" (Baader und Sager 2010, S. 242).

Auch wenn die Bedeutung kindlicher Selbstbildungsprozesse bereits zuvor im Rahmen reformpädagogischer Konzepte (an-)erkannt worden war (Aden-Grossmann 2011), so war es die Kinderladenbewegung, der es nachhaltig gelang, ihre pädagogischen und bildungspolitischen Anliegen in Fachdiskurse einzubringen und ihre Ansätze beispielsweise über Modellprojekte (vgl. Schmid 2008) auch über die Kinderläden hinaus zu verbreiten. Damit trug sie zu einer ,Modernisierung des Vorschulbereichs von unten“ bei (Baader 2014, S. 431).

\subsection{Entwicklung und Verbreitung}

Ausgehend von verschiedenen Groß- und Universitätsstädten Deutschlands verbreitete sich das Modell des Kinderladens zunehmend auch in Kleinstädte und den ländlicheren Raum. Dabei standen mit dem zeitlichen Voranschreiten meist weniger die politischen Bestrebungen (Palm und Winkler 2019 [1996]; Silvester 2009), als vielmehr die Nachfrage nach Betreuungsplätzen im Zuge der stetig ansteigenden Frauenerwerbsarbeitsquote sowie der elterliche Wunsch nach außerfamilialen Sozialkontakten für ihre Kinder und nach alternativen pädagogischen Konzepten (bspw. Natur- und Waldkindergärten, Waldorfeinrichtungen, integrativen Einrichtungen oder bilingualen Konzepten) im Vordergrund (Baader 2014). Mit ihrem Innovationspotenzial füll(t)en Elterninitiativen Nischen, die vom Bereich der frühen Bildung, Betreuung und Erziehung (FBBE) nicht, oder nicht ausreichend, bedient wurden oder werden; als weitere Beispiele hierfür lassen sich u.a. Krippenplätze für Kinder unter drei Jahren, oder Betreuungseinrichtungen in ländlichen Regionen anführen (Aden-Grossmann 2011; Baader 2020).

Seit 2006 berücksichtigt das Statistische Bundesamt Elterninitiativen in seiner jährlichen Erhebung der Kinder- und Jugendhilfestatistik als spezifische Trägerform innerhalb der Gruppe der freien Träger. Aktuell sind von den bundesweit 57.594 verzeichneten Kindertageseinrichtungen 4188 Einrichtungen Elterninitiativen, was etwa 7,3\% Prozent entspricht (DESTATIS 2020). Überdurchschnittlich viele Elterninitiativen gibt es in den Stadtstaaten Bremen (25\%) und Berlin (21\%); in NordrheinWestfahlen $(11 \%)$, Niedersachsen $(8,5 \%)$ und Schleswig-Holstein (8\%) liegt ihr Anteil ebenfalls über dem Bundesdurchschnitt (ebd.).

\subsection{Professionalisierungsprozesse}

Ein Grund für den erfolgreichen Fortbestand der Elterninitiativen kann u.a. auch darin vermutet werden, dass sie sich institutionalisiert (Reyer und Müller 1992) und professionalisiert haben, und heute auf solide selbstgeschaffene Unterstützungsstrukturen und Netzwerke zurückgreifen können (Baader und Riechers 2018). Gebündelt werden diese in der 1986 gegründeten Bundesarbeitsgemeinschaft Elterninitiativen e. V. (BAGE), der bundesweit 27 Dach- und sechs Landesverbände angeschlossen sind, die Anlaufstellen für bestehende Elterninitiativen bieten und engagierte Eltern bei der Gründung neuer Einrichtungen unterstützen (BAGE 2019). Wie wichtig diese Unterstützungsstrukturen sind, wird insbesondere mit Blick auf die Vielzahl 
an Anforderungen, die heute an Kindertagesbetreuungseinrichtungen herangetragen werden, ersichtlich.

Während sich durch die Implementation neuer Gesetze und Richtlinien der Arbeits- und Verwaltungsaufwand in den Einrichtungen stetig erhöht, lastet im Zuge gesamtgesellschaftlicher Entwicklungen heute auf allen Eltern ein zunehmender Effektivitätsdruck, der sich auf ihre Bereitschaft und Möglichkeiten zum Engagement in den Vereinen ${ }^{4}$ auswirken kann (Baader und Bollig 2019, Baader und Riechers 2018; Freiling 2003; Silvester 2009). Damit stehen die Vereine vor der Herausforderung, die praktische Organisations- und Alltagsgestaltung bei gleichzeitiger Zunahme der Anforderungen an die Einrichtungen der FBBE und der Abnahme der elterlichen Ressourcen für ehrenamtliche Arbeit zu gewährleisten. Beobachten lassen sich infolgedessen Strukturveränderungen (Vereinsumstrukturierungen, Zusammenschlüsse zu größeren Trägerverbänden) sowie Professionalisierungsprozesse (das Outsourcen von Elterndiensten an externe Dienstleistungsunternehmen) oder das Nutzen der Dienstleistungsangebote der Dachverbände (Beratungsangebote, etwa zur Umsetzung arbeitsrechtlicher Bestimmungen, Unterstützung bei der Buchhaltung u. Ä.).

\section{Qualitative Perspektiven auf „Inklusive Elterninitiativen“}

Zur Diskussion der eingangs aufgeworfenen Frage nach dem Zusammenhang des pädagogisch-konzeptionellen und organisationalen Partizipationsverständnisses der Elterninitiativen sowie damit in Verbindung stehenden In- und Exklusionsprozessen, wird sich im Folgenden auf den Datenkorpus und die Ergebnisse der Studie „Inklusive Elterninitiativen“ (IKE) bezogen. Auf Basis heuristischer Annahmen - die sich durch den vorangehend dargestellten historischen Entstehungskontext und die spezielle Organisationsform der Elterninitiativen konturieren lassen - arbeitete IKE mit der These, dass sich bei dieser speziellen Trägerform spezifische Ausprägungen und Umsetzungsformen von Inklusion finden lassen. Ziel des Projekts war es, einen explorativen Einblick in die Handlungspraxen und Konstruktionsleistungen, die Elterninitiativen als bildungs- und sozialpolitisch relevante Akteure in den Inklusionsdiskurs einbringen, zu erlangen.

\subsection{Methodischer Zugang}

Grundlage des Erhebungs- und Auswertungsverfahrens bildet das Expert*innneninterview nach Meuser und Nagel (2005a [1991]), die erstmals eine methodologische Eingrenzung des Expert*innenstatus vorgenommen und strukturierte Auswertungsschritte für den Umgang mit Expert*inneninterviews formuliert haben. Dabei ist Expert*in ein relationaler Status, den Forschende einer Person mit einer bestimmten Position in einem konkreten Wissens- und Handlungsgebiet zuschreiben,

\footnotetext{
4 Auch heute noch leisten Eltern ehrenamtliche Dienste in den Elterninitiativen; als Vereinsmitglieder tragen sie die Hauptverantwortung für die Einrichtung und fungieren als Arbeitgeber*innen der pädagogischen Fachkräfte (Aden-Grossmann 2011; BAGE 2019).
} 
die somit ,,selbst Teil des Handlungsfeldes [ist], das den Forschungsgegenstand ausmacht" (ebd., S. 73). In Anlehnung an Gorden (1975) definieren Meuser und Nagel (2005b) Expert*innen als aktive Partizipant*innen, die ihr Expert*innenwissen über berufliches oder ehrenamtliches Engagement erwerben, und damit ,typische Problemtheorien, Lösungswege und Entscheidungsstrukturen“ (ebd., S. 264) ihres institutionellen Kontextes und Handlungsfeldes repräsentieren.

Im Hinblick auf das Expert*innenwissen differenzieren Meuser und Nagel zwischen zwei unterschiedlichen Wissensformen. Beim Betriebswissen gelten die befragten Expert*innen als Zielgruppe der Untersuchung; sie gewähren Einblicke in ihr eigenes Handlungsfeld und ermöglichen somit einen Zugang zu ihren impliziten Handlungsmaximen und institutionalisierten Prozessen (ebd. sowie ebd. 2005a [1991]). Bei der Ermittlung von Kontextwissen steht dagegen die Informationsgewinnung über die Kontextbedingungen des Handelns der Zielgruppe im Fokus. Die Expert*innen gelten hier als ,eine zur Zielgruppe komplementäre Handlungseinheit““ (ebd. 2005a [1991], S. 75; H.i.O.), die durch die von ihnen ausgehenden Effekte und Normierungen die Kontextbedingungen des zu erforschenden Handlungsfeldes mitbestimmt. ${ }^{5}$

In Erweiterung der von Meuser und Nagel vorgenommenen Differenzierung verweisen Przyborski und Wohlrab-Sahr (2014) zudem auf den Aspekt des Deutungswissens, der „die Deutungsmacht der Experten als Akteure“ (ebd., S. 121) in besonderer Weise hervorhebt und ihre aktive Beteiligung ,,an der Etablierung und Durchsetzung von Deutungen“ beleuchtet (ebd., S. 120).

Meuser und Nagel (2005a [1991]) beschreiben die von ihnen entwickelte Auswertungsstrategie als „Entdeckungsstrategie“ (ebd., S. 81), die u. a. genutzt wird, um Wissen über die impliziten Funktionslogiken sozialer Systeme zu erlangen, Einblicke

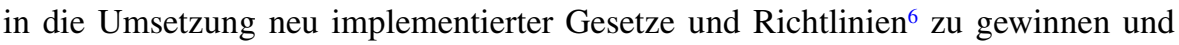
organisationsspezifische Barrieren ebenjener offenzulegen (ebd. sowie ebd. 2005b).

\subsection{Feldzugang, Sample und Erhebung}

Der Zugang zu den niedersächsischen Elterninitiativen erfolgte über das Netzwerk der Landesarbeitsgemeinschaft Elterninitiativen Niedersachsen/Bremen e. V. (lagE). Dabei wurden nicht nur Elterninitiativbetreuungseinrichtungen selbst, sondern auch die niedersächsischen Dachverbände in den Blick genommen, die als Beratungsstellen, Verwaltungsdienstleister und politische Interessenvertretungen der Elterninitiativen fungieren. Das methodologische Vorgehen wurde daher so gewählt, dass sich beide Ebenen - die der pädagogischen Praxis und der politischen Interessenvertretung - erfassen lassen.

\footnotetext{
5 Die Analyse von Kontextwissen dient dem Abstecken des Sachverhalts sowie der Generierung von Themen und Hypothesen; das Ziel der Analyse von Betriebswissen ist dagegen auf die theoretische Generalisierung gerichtet (Meuser und Nagel 2005a [1991]).

6 Im Falle von IKE bezieht sich dies etwa auf die Umsetzung des nun seit 2009 rechtlich verankerten Anspruchs auf ,inklusive“ Bildung (UN-BRK); aber auch auf die Ausgestaltung des Kinderrechts auf Beteiligung, Mitsprache und Mitgestaltung (UN-KRK) sowie des Wunsch- und Wahlrechts der Eltern (SGB VIII).
} 
Der Auswahl des Samples gingen zunächst eine Website- und Dokumentenanalyse sowie beratende Gespräche auf der Dachverbandsebene voraus. Da sie von den übergeordneten regionalen Verbänden hin zu den ihnen zugehörigen Mitgliedseinrichtungen verdichtet wurde, konnte die Expertise der Verbände für die Gewinnung einer möglichst heterogenen Gesamtstichprobe an Einrichtungen genutzt werden. Befragt wurden letztlich Mitarbeitende und/oder Vorstandsmitglieder aus allen sieben niedersächsischen Dachverbänden sowie pädagogische Fachkräfte und Einrichtungsleitungen aus insgesamt 21 Elterninitiativeinrichtungen (Riechers und Baader 2021).

Aufgrund der Annahme, dass sich auf der Verbandsebene sowohl Kontext- als auch Betriebswissen findet, wohingegen der Fokus der Einrichtungsebene stärker durch das Betriebswissen geprägt ist, wurden beide Ebenen multiperspektivisch betrachtet $^{7}-$ Ziel war sowohl die Hypothesengewinnung als auch die theoretische Generalisierung.

Über leitfadengestützte Expert*inneninterviews wurden die Vereinsselbstbeschreibung (Entstehung, Entwicklung, Struktur/Organisation, Selbstverständnis und Schwerpunkte) und die vereinsspezifischen Sichtweisen und Handlungspraxen bzgl. der Bereiche Kindheit/kindliche Selbstbestimmung, Heterogenität/Vielfalt/Inklusion sowie Partizipation/Beteiligungsstrukturen erfragt.

\subsection{Auswertung}

Das transkribierte Datenmaterial (Audioaufzeichnungen) wurde programmunterstützt aufbereitet. Mittels einer ersten - an die inhaltlich strukturierende Inhaltsanalyse (Kuckartz 2016) angelehnten - induktiven Kategoriebildung wurde der Datenkorpus vorstrukturiert und die im Sinne der Forschungsfragen relevanten Textpassagen markiert. Die anschließende Analyse erfolgte entlang der strukturierten Auswertungsschritte ${ }^{8}$ nach Meuser und Nagel (2005a [1991]). Dabei wurden die Dachverbands- und Einrichtungsebene zunächst voneinander getrennt untersucht und die hier jeweils gewonnenen Erkenntnisse erst in einem letzten kontrastierenden Analyseschritt zusammengeführt. Zudem wurde die Einrichtungsebene ebenfalls in sich untergliedert analysiert, um themen- und fallspezifische Auswertungsoptionen zwischen autonomen Elterninitiativvereinen und solchen, die sich in größeren Trägerverbänden zusammengeschlossen haben, zu ermöglichen. ${ }^{9}$

\footnotetext{
7 Deutungswissen findet sich auf beiden Erhebungsebenen.

8 Diese beinhalten die Paraphrasierung relevanter Textstellen und die induktive Kategoriebildung auf Basis der Paraphrasen sowie einen anschließenden thematischen Vergleich - anhand der Zusammenstellung ähnlicher Passagen und das Herausarbeiten erster Relevanzstrukturen - und schließlich das Abzielen auf die Abstraktionsebene, über die von den Einzelinterviews losgelöste Systematisierung von Relevanzstrukturen und Deutungsmustern.

9 Dem Netzwerk der lagE sind in Niedersachsen drei Kontakt- und Beratungsstellen (Dachverbände ohne eigene Trägerschaft, die autonome Elterninitiativvereine vertreten und mit Dienstleistungen unterstützen) sowie vier Verbundträger (Dachverbände mit eigener Trägerschaft, die als ein eingetragener Verein unterschiedlich viele Elterninitiativeinrichtungen betreiben) angeschlossen. Im prozentualen Verhältnis der durch sie vertretenen Einrichtungen liegt die Gewichtung der lagE-Mitglieder bei $79 \%$ autonomen Elterninitiativvereinen zu $21 \%$ Einrichtungen in Verbundträgerschaft. Das Sample der Studie IKE umfasst
} 


\section{Inklusion und Partizipation aus Sicht niedersächsischer Elterninitiativvereine}

Das Projekt IKE zielte darauf ab, Kontextbedingungen von Inklusion über damit verbundene soziale Konstruktionsleistungen am Beispiel der Trägerform Elterninitiative $\mathrm{zu}$ erforschen. Der Forschungsanlage lag ein weites Inklusionsverständnis zugrunde (Hinz 2010), das das Konzept Inklusion als einen gesellschaftlichen und systemischen (Reflexions-)Ansatz fasst (Sander 2004) und somit nicht auf die nähere Beleuchtung additiver (sonderpädagogischer) Fördermaßnahmen oder -settings fokussiert. In Anschluss an Budde und Hummrich (2015) wird zudem davon ausgegangen, dass der Inklusionsbegriff aus erziehungswissenschaftlicher Perspektive untauglich ist, sofern er nicht mit seinem Komplementärbegriff - der Exklusion - zusammengedacht wird. Die im Folgenden exemplarisch veranschaulichten Ergebnisse der IKE-Studie werden daher perspektivisch entlang des rekonstruierten Nachvollzugs der reflexiven Bearbeitung des Spannungsfelds von In- und Exklusion durch die Elterninitiativvereine ${ }^{10}$ strukturiert und durch die Bezugnahme auf verschiedene Konzepte von Inklusion theoretisch gerahmt.

\subsection{Inklusion als Grundhaltung}

Den zentralen Ausgangspunkt bildet die normative Kategorie Inklusion als Grundhaltung, die sich in allen Interviews rekonstruieren ließ. Elterninitiativvereine verweisen auf die individuellen Besonderheiten aller Menschen, die es anzuerkennen und zu berücksichtigen gilt. Dabei rekurrieren sie auf eine Grundhaltung, die auf den allgemeinen Menschen- und Kinderrechten fußt, und leiten daraus ihren Selbstanspruch ab, jeden Menschen so, „wie er ist, mit dem was er kann“, anzunehmen und Praxen zu schaffen, die es ermöglichen, dass jede*r teilhaben kann. Dieses grundsätzliche Verständnis vom Menschen und der Gleichwertigkeit seiner individuellen Vielfalt wird von ihnen als „Elterninitiativgedanke“ beschrieben, der als Kondensat ihres Einrichtungsselbstverständnisses bezeichnet werden kann. Elterninitiativen setzen somit ihr Inklusionsverständnis mit ihrem Selbstverständnis und ihrem Menschen- bzw. Kindbild gleich. Aufgrund dessen wird Inklusion bei vielen Vereinen konzeptionell auch nicht als gesonderter Punkt aufgeführt, sondern findet sich implizit in ihrem Selbstanspruch, alle Kinder - unabhängig von Etikettierungen - als Persönlichkeiten mit individuellen Bedürfnissen und Bedarfen zu verstehen (Riechers und Baader 2021).

In ihrer Ablehnung kategorialer Zuschreibungen und der Betonung der individuellen Vielfalt aller Menschen lässt sich ein Inklusionsverständnis identifizieren, das mit Katzenbach (2015) gelesen auf eine Dethematisierung von Unterschieden zuguns-

auf der Einrichtungsebene ein Verhältnis von $62 \%$ autonomen Elterninitiativen zu $38 \%$ Einrichtungen in Verbundträgerschaft.

10 Auf die in 3.3 dargelegte Differenzierung der Ebenen Dachverbände und Einrichtungen sowie autonome Einrichtungen und Einrichtungen in Verbundträgerschaft wird in dieser Darstellung weitestgehend verzichtet. Die Bezeichnung die Elterninitiativen schließt hier insoweit alle dieser Ebenen und Formen ein, als dass in den dargelegten empirischen Inhalten keine signifikant differierenden Praxen oder Ansichten vorliegen. Andernfalls werden diese an den entsprechenden Stellen gesondert betont. 
ten der Betonung der Gleichwertigkeit aller Menschen in ihrer Unterschiedlichkeit abzielt. In Anschluss an Mai-Anh Bogers Theorie der trilemmatischen Inklusion ${ }^{11}$ (2015) kann ihre Grundhaltung damit als ein transnormalistisches Inklusionsverständnis gedeutet und auf der Achse Normalisierung und Dekonstruktion verortet werden (siehe dazu auch Gestenberg et al. 2021).

\subsection{Inklusion als Prozess der aktiven Herstellung}

Wird diese Grundhaltung auf die Ebene der pädagogischen Praxis transferiert, so wird Inklusion hier folgelogisch als Ansatz begriffen, der den Ausgang des pädagogischen Handelns am jeweiligen Kind und seinen individuellen und veränderlichen Bedürfnissen festzumacht. Mit Brunner (2018) kann die Handlungsorientierung der pädagogischen Fachkräfte der Elterninitiativen damit vorrangig als ,,bedürfnisorientiert[.] entwicklungsbegleitend[.]“ (ebd., S. 181; H.i. O.) charakterisiert werden; worunter Brunner - in Anlehnung an den Normalismusbegriff nach Link (2013) eine transnormalistisch-dekonstruktivistische Strategie fasst, der zufolge kategorisierende Zuschreibungen für das pädagogische Handeln irrelevant werden, da alle Kinder gemäß ihrer situativen Bedürfnisse entsprechende Begleitung und Förderung erhalten (Brunner 2018).

Inklusion kann damit als Prozess der aktiven Herstellung rekonstruiert werden, der von der pädagogischen Ebene auf die organisationale Ebene verweist. Elterninitiativen treten dafür ein, nicht das Kind dem System „passig“ machen zu wollen, sondern Bildungssettings und pädagogische Angebote so zu gestalten, dass sie allen Kindern gerecht werden können. Dieser Selbstanspruch der Elterninitiativen gerät jedoch mitunter an seine Grenzen, was den Vereinen insbesondere in der Diskrepanz zwischen den Rahmenbedingungen für die Betreuung von sogenannten ,Integrationskindern' und den tatsächlichen Realgruppen(alltags)bedingungen bewusstwird. Einerseits streben Elterninitiativen an, Vielfalt zu leben, ohne Differenzmerkmale explizit thematisieren zu müssen. Andererseits bleibt bei der derzeitigen rahmenrechtlichen Umsetzung von ,inklusiver' Betreuung jedoch die Notwendigkeit bestehen, Differenzmerkmale hervorzuheben, um auf Basis einer Diagnostik die Finanzierung entsprechender Betreuungsbedingungen abzusichern. Damit verweisen sie auf die dilemmatische Koppelung der Gewährung finanzieller Mittel für ,inklusive ‘ Betreuungsbedingungen an individuumsbezogene diagnostische Anerkennungsprozesse, die aus wissenschaftlicher Perspektive bereits unter der Bezeichnung Etikettierungs-Ressourcen-Dilemma erörtert wurde (siehe dazu Kornmann 1994). Dieser Problematik begegnen die Vereine - an die Typenbildung von Brunner (2018) anschließend - mit einer, ebenfalls Link entlehnten, ,flexibel-normalistische[n] Strategie“ (ebd., S. $35 \mathrm{ff}$.), die als pragmatisch-handlungspraktischer und ,strukturgebundene[r]“" Lösungsansatz verstanden werden kann (ebd., S. 181; H.i. O.). Entgegen ihres normalistisch-dekonstruktivistischen Inklusionsverständnisses nehmen somit

\footnotetext{
11 Boger (2015) beschreibt Inklusion über drei Basissätze, die sie in Form eines gleichwinkligen Dreiecks visualisiert: Inklusion als Empowerment, Inklusion als Normalisierung und Inklusion als Dekonstruktion. Dabei können jeweils zwei dieser Basissätze zu einer Achse kombiniert werden, wodurch der nun einzelnstehende Basissatz als uneinlösbar markiert wird.
} 
auch Elterninitiativen Dichotomisierungen zwischen Integrations- und Regelgruppen oder -kindern vor; diese Differenzierung ist jedoch strukturell bedingt und verweist vorrangig auf die institutionell-organisatorische, und weniger auf die pädagogische Ebene.

\subsection{In- und Exklusion als wechselseitig bedingte Kategorien}

Um entgegen der vorangehend dargelegten pragmatisch-dichotomisierenden Praktik ihr Selbstverständnis und ihre Handlungspraxen in Bezug auf Inklusion miteinander in Übereinstimmung zu bringen, nutzen Elterninitiativvereine eine Strategie, die zentral auf ihre entstehungsgeschichtlichen Wurzeln und Kernwerte - Solidarität und Partizipation - verweist (Baader und Riechers 2018). Statt angesichts unzureichender Rahmenbedingungen die „Hände in den Schoß [zu] legen “, nutzen die Vereine die Kompetenzen, Netzwerke und Ressourcen ihrer Mitglieder, um in produktiver Kombination dessen, was jede*r individuell einbringen kann, in Eigenengagement tragende Unterstützungsstrukturen auszubilden und für ihre Gruppen Bedingungen zu schaffen, die ihnen Spielräume für dekategoriale Inklusionspraxen ermöglichen. ${ }^{12}$ In diesem Sinne kreieren sie für sich strukturelle Freiräume, in denen sie versuchen, sich z.T. gezielt von rahmenrechtlich bedingten Etikettierungs- und Normierungspraxen abzugrenzen. Mit Boger (2015) lässt sich diese trägerspezifische Ausdeutung von Inklusion der Achse Dekonstruktion und Empowerment zuordnen, die sich als widerständige Haltung der Normalisierungsverweigerung charakterisieren lässt (Gerstenberg et al. 2021).

Zugleich begeben sich die Elterninitiativen damit jedoch auch in eine Position der selbstgewählten Exklusion und ,Selbstverbesonderung“ (Boger 2016, o. S.). Elterninitiativen sind in ihrem entstehungsgeschichtlichen Kontext als Selbsthilfeorganisationen zu verstehen, die sich über einen Zusammenschluss von Gleichgesinnten konstituier(t)en, was zu einem erschwerten Zugang bzw. Ausschluss von Anderen führen kann. ${ }^{13}$ Die besonderen Bedingungen, die Elterninitiativeinrichtungen kennzeichnen - kleine Einrichtungs- und Gruppengrößen, ein oftmals besonders guter Betreuungsschlüssel und hohe pädagogische Qualität, eine enge und kooperative Partnerschaft von Eltern und pädagogischen Fachkräften ${ }^{14}$ - führen dazu, dass sich Inklusion im Inneren gut und gemäß ihres dekategorialen Selbstanspruchs verwirklichen lässt. Daraus erwächst gleichwohl die Annahme, dass sich diese exklusiv guten Bedingungen nur darüber aufrechterhalten lassen, dass sich die Vereine - bewusst wie unbewusst - nach außen hin abgrenzen.

Die IKE-Erhebungen können aufzeigen, dass Elterninitiativen sich dieses Spannungsfelds durchaus bewusst sind. Zum Prozess der (selbst-)reflexiven Auseinander-

\footnotetext{
12 So kam und kommt es bspw. vor, dass sich die Elternschaft als basisdemokratische Gemeinschaft dafür entscheidet, in finanzielle Vorleistung zu gehen, um einzelnen Kindern und Familien entsprechende Betreuungsbedingungen zu eröffnen, ohne dass diese zwangsläufig an Zuschreibungsprozesse geknüpft werden (müssen).

13 Diese Problematik besteht seit den entstehungsgeschichtlichen Anfängen der Elterninitiativen und kann damit als „historische Pfadabhängigkeit“ (Baader und Bollig 2019, S. 146) bezeichnet werden (siehe dazu auch Baader 2008, 2020; Iseler 2010; Palm und Winkler 2019 [1996]).

14 Siehe dazu bspw. Aden-Grossmann (2011) sowie Baader und Riechers (2018).
} 
setzung mit der Thematik Inklusion gehört für sie daher zwangsläufig auch, sich mit möglichen Exklusionsmechanismen, etwa strukturellen Zugangshemmnissen oder teilhabebeschränkenden Handlungspraxen der eigenen Einrichtung, auseinanderzusetzen (siehe dazu auch Baader 2020 sowie Riechers und Baader 2021). Dabei kann der hier dargelegte Selbstanspruch, sich mit dem Zusammenspiel verschiedener Strukturkategorien zu befassen, um darin eingeschriebene Formen von Benachteiligung bearbeiten zu können, mit Budde und Hummrich (2015) als ein reflexives Inklusionsverständnis gelesen werden. ${ }^{15}$

\section{Partizipation - Teilhabe als Ausschlussfaktor?}

Ein zentraler Faktor für die Funktionalität und den Fortbestand der Elterninitiativen besteht nach wie vor darin, dass sich die Elternschaft in den Vereinen einbringt. ${ }^{16}$ Damit wird das elterliche Engagement zum Kristallisationspunkt des Spannungsfelds von In- und Exklusion: Elterninitiativen wollen für jede*n zugänglich sein, stehen jedoch zugleich vor der Problematik, dass nicht alle Eltern gleichermaßen über die Zeit, Ressourcen und - je nach Aufgabenfeld - ggf. Kompetenzen verfügen, sich entsprechend in den Vereinen einbringen zu können (Riechers und Baader 2021).

Aus den Ergebnisse der IKE-Studie lassen sich im Zusammenspiel der Ebenen Betriebs-, Kontext- und Deutungswissen verschiedene Deutungsmuster herausarbeiten, anhand derer sich die Persistenz der Betonung der Elternbeteiligung durch die Vereine konturieren lässt.

Heutige Elterninitiativen verstehen Partizipation als Grundelement ihrer speziellen Trägerform, das für sie sowohl eine organisationale Notwendigkeit darstellt, die den Fortbestand der Vereine sichert, als auch als ein pädagogisches Prinzip, das konzeptionell verankert ist und in der alltäglichen Praxis durch alle Beteiligten gelebt wird. Welche Bedeutung dem Prinzip der Partizipation zuteilwird, drückt sich im folgenden Zitat eines Vorstandsmitglieds aus:

Wir möchten gerne eine wertschätzende Pädagogik haben und denken eben, das muss sich auch in den Strukturen ausdrücken [...,] weil wir denken, wenn sich die Struktur der Zusammenarbeit ändern würde, dann würde das auch Auswirkungen haben auf den Umgang miteinander in der Einrichtung und möglicherweise auch auf die Pädagogik.

\footnotetext{
15 Dies beziehen Elterninitiativen auf die kritisch-reflexive Bearbeitung ihrer eigenen Strukturen, aber - insbesondere auf der Dachverbandsebene - auch auf das aktive Einbringen ihres Deutungswissens und ihrer grundlegenden Strukturkritik auf Kommunaler, Landes- und Bundesebene. In Anschluss an Boger (2015) findet sich hier somit auch die Verortung auf der Achse Empowerment und Normalisierung wieder, in deren Sinne sich die Elterninitiativen als bildungs- und sozialpolitisch relevante Akteure für das Recht Aller auf Teilhabe engagieren.

16 Dabei ergeben sich z. T. Differenzierungen zwischen den autonomen kleinen Elterninitiativvereinen, die in noch höherem Maße von der elterlichen Mitarbeit und Verantwortungsübernahme abhängig sind, und den Einrichtungen in Verbundträgerschaft, die Elternarbeit weit häufiger als Austausch mit und Leistungen für Eltern, denn als Engagement der Eltern für den Verein, definieren.
} 
Das gemeinsame Partizipieren am Vereinsgeschehen - bei dem sich Eltern und pädagogische Fachkräfte jeweils bereichsspezifisch kooperierend für die Elterninitiativeinrichtung verantwortlich zeigen - wird hier in Stringenz zur pädagogischen Konzeption gesetzt. Kindern, die ihre pädagogischen Fachkräfte als gleichberechtigte Teammitglieder und ihre Eltern als Teil der Aushandlungsprozesse innerhalb der Vereine und als Mitgestaltende selbiger erleben, wird es aus Sicht der Elterninitiativen in besonderer Weise ermöglicht, „Demokratie zu erleben“. Damit erachten Elterninitiativen die Reduktion der elterlichen Mitarbeit im Verein als eine Gefahr für ihre Einrichtungsidentität, die sich u.U. bis auf die Ebene des Pädagogischen auswirken könnte.

Einerseits würde sich eine Abkehr von der starken Betonung der elterlichen Beteiligung ggf. positiv auf die Vielfalt der Elternschaft auswirken, da sich die vorangehend beschriebene zugangshemmende Wirkung der Elterndienste so abschwächen könnte. Andererseits besteht die Annahme, dass mit einer (weiteren) Reduktion der Elternbeteiligung - bspw. durch das fortschreitende Outsourcen von Elterndiensten, oder der Abgabe von Verwaltungsposten an eine hauptamtliche Geschäftsführung eine mögliche Zunahme des Dienstleistungsgedankens ${ }^{17}$ in der Elternschaft befördert werden könnte. Dies erachten Elterninitiativen vor allem hinsichtlich der inkludierenden Wirkung, die sie den Elterndiensten zuschreiben, als problematisch. So kann die IKE-Studie rekonstruieren, dass insbesondere durch die aktive partizipative Zusammenarbeit in den Einrichtungen eine Nähe entsteht, die den Eltern Einblicke in vielfältige Lebenssituationen und individuelle familiäre Bedürfnisse gewährt, was sich prägend auf ihren Umgang mit Vielfalt auswirken und zu mehr Offenheit und Solidarität beitragen kann.

Eine weitere Befürchtung schließt an Überlegungen zur Erhöhung der elterlichen Vielfalt in den Einrichtungen - z. B. über eine gezielte diversitätssensible Zusammensetzung im Rahmen des Aufnahmeprozederes - an. Elterninitiativen befassen sich reflexiv mit der Wirkmächtigkeit ihrer eigenen, je einrichtungsspezifischen Zugangshemmnisse und betonen vielfach explizit ihren Wunsch nach mehr Vielfalt. ${ }^{18}$ Zugleich besteht jedoch auch die Sorge, wie sich eine heterogenere Elternschaft auf das für sie so charakteristische Element des Elternengagements auswirken könnte (siehe auch Baader 2020). Damit steht der Wunsch nach einer heterogeneren Elternschaft gleichzeitig der Annahme gegenüber, dass dies ggf. zu Unruhen in der Elternschaft führen könnte, ${ }^{19}$ was als Risikofaktor hinsichtlich des Zusammenhalts und damit für die Elternbeteiligung im Verein - und letztlich für dessen Identität und Fortbestand - empfunden wird.

Diesem Dilemma wird seitens der Vereine mitunter dadurch begegnet, dass kategoriale Zuschreibungen bewusst dekonstruiert werden: Sofern Heterogenität vor allem als individuelle persönliche Vielfalt verstanden wird, kann sich auch eine

\footnotetext{
17 Siehe hierzu auch Freiling (2003), Iseler (2010), Palm und Winkler (2019 [1996]), Silvester (2009).

18 Bspw. in Bezug auf Herkunft der Familien, Einkommen/soziale Schicht, Bildungsstand, Familienmodell o. Ä.

19 Etwa durch das Aufeinandertreffen unterschiedlicher Vorstellungen und Praktiken; bspw. in Bezug auf Erziehungsvorstellungen oder bzgl. der Bereitschaft zur Mitarbeit im Verein.
} 
Einrichtung, die ihre Elternschaft in Bezug auf einige Merkmale ${ }^{20}$ als homogen beschreibt, mit dem Fokus auf die persönliche Einzigartigkeit aller Menschen in ihrem Selbstkonzept dennoch als vielfältig begreifen. Inklusion bleibt damit ein standpunktbezogener Verhandlungsgegenstand.

Funding Open Access funding enabled and organized by Projekt DEAL.

Open Access Dieser Artikel wird unter der Creative Commons Namensnennung 4.0 International Lizenz veröffentlicht, welche die Nutzung, Vervielfältigung, Bearbeitung, Verbreitung und Wiedergabe in jeglichem Medium und Format erlaubt, sofern Sie den/die ursprünglichen Autor(en) und die Quelle ordnungsgemäß nennen, einen Link zur Creative Commons Lizenz beifügen und angeben, ob Änderungen vorgenommen wurden.

Die in diesem Artikel enthaltenen Bilder und sonstiges Drittmaterial unterliegen ebenfalls der genannten Creative Commons Lizenz, sofern sich aus der Abbildungslegende nichts anderes ergibt. Sofern das betreffende Material nicht unter der genannten Creative Commons Lizenz steht und die betreffende Handlung nicht nach gesetzlichen Vorschriften erlaubt ist, ist für die oben aufgeführten Weiterverwendungen des Materials die Einwilligung des jeweiligen Rechteinhabers einzuholen.

Weitere Details zur Lizenz entnehmen Sie bitte der Lizenzinformation auf http://creativecommons.org/ licenses/by/4.0/deed.de.

\section{Literatur}

Aden-Grossmann, W. (2011). Der Kindergarten: Geschichte - Entwicklungen - Konzepte. Weinheim, Basel: Beltz.

Aden-Grossmann, W. (2019). Monika Seifert - Gründerin der Antiautoritären Erziehungsbewegung. In K. Bock, N. Göddertz, F. Heyden \& M. Mauritz (Hrsg.), Zugänge zur Kinderladenbewegung (S. 9-26). Wiesbaden: Springer VS.

Albers, T., Bruck, S., Even, J., Lüpke, S., Thomas, S., \& Tremel, H. (2011). Kitas als Türöffner. Integrative Tageseinrichtungen für Kinder als Schlüssel zur gleichberechtigten Teilhabe. Hannover: lagE e. V., Leibniz Universität Hannover.

Baader, M. S. (2007). Erziehung gegen Konkurrenzkampf und Leistungsprinzip als gesellschaftsverändernde Praxis. 68 und die Pädagogik in kultur- und professionsgeschichtlichen Perspektiven 1965-1975. Zeitschrift für pädagogische Historiographie, 2, 23-29.

Baader, M. S. (2008). Von der sozialistischen Erziehung bis zum buddhistischen Om. Kindeläden zwischen Gegen- und Elitekulturen. In M. S. Baader (Hrsg.), , Seid realistisch, verlangt das Unmögliche! “ Wie 68 die Pädagogik bewegte (S. 16-35). Weinheim, Basel: Beltz.

Baader, M. S. (2009). Öffentliche Kleinkindererziehung in Deutschland im Fokus des Politischen. Von den Kindergärten 1848 zu den Kinderläden in der 68er Bewegung. In J. Ecarius, C. Groppe \& H. Malmede (Hrsg.), Familie und öffentliche Erziehung. Theoretische Konzeptionen, historische und aktuelle Analysen (S. 267-289). Wiesbaden: VS.

Baader, M. S. (2011). „An der großen Fensterscheibe sollen sich die Kinder von innen und die Passanten von außen die Nase platt drücken“ Kinderläden, Kinderkulturen und Kinder als Akteure im öffentlich-städtischen Raum seit 1968. In M.S. Baader \& U. Herrmann (Hrsg.), 68 - Engagierte Jugend und Kritische Pädagogik. Impulse und Folgen eines kulturellen Umbruchs in der Geschichte der Bundesrepublik (S. 232-251). Weinheim, München: Juventa.

Baader, M. S. (2014). Die reflexive Kindheit. In M. S. Baader, F. Eßer \& W. Schröer (Hrsg.), Kindheiten in der Moderne. Eine Geschichte der Sorge (S. 414-455). Frankfurt a.M.: Campus.

Baader, M. S. (2020). Von der Antiautorität zur Diversität. Soziale Differenzen in Kinderläden und Elterninitiativen von den 1970er Jahren bis heute. Geschichte und Gesellschaft. Zeitschrift für Historische Sozialwissenschaft, 46(H. 2), 200-230.

${ }^{20}$ Etwa primär akademisch geprägt und häufig gut situiert (dazu auch Baader 2020). 
Baader, M.S., \& Bollig, S. (2019). „Elternbeiträge“: Eltern als individuelle wie kollektive Wohlfahrtsproduzenten im Feld der frühen Bildung, Betreuung und Erziehung. Zeitschrift für Soziologie der Erziehung und Sozialisation, 39(H. 2), 134-151.

Baader, M.S., \& Riechers, K. (2018). Kitas in Selbstorganisation: Elterninitiativen gestern - heute - morgen. Kita aktuell, 26, 224-226.

Baader, M. S., \& Sager, C. (2010). Die pädagogische Konstitution des Kindes als Akteur im Zuge der 68erBewegung. Diskurs Kindheits- und Jugendforschung, 3, 255-269.

BAGE - Bundesarbeitsgemeinschaft Elterninitiativen e. V. (2019). Gründungsleitfaden für Elterninitiativen (2. Aufl.). Berlin: BAGE.

Berliner Kinderläden (1970). Antiautoritäre Erziehung und sozialistischer Kampf. Köln, Berlin: Kiepenheuer \& Witsch.

Bock, K., Göddertz, N., Heyden, F., \& Mauritz, M. (2019). Statt eines Nachworts. In K. Bock, N. Göddertz, F. Heyden \& M. Mauritz (Hrsg.), Zugänge zur Kinderladenbewegung (S. 389-429). Wiesbaden: Springer VS.

Boger, M.-A. (2015). Theorie der trilemmatischen Inklusion. In I. Schnell (Hrsg.), Herausforderung Inklusion - Theoriebildung und Praxis (S. 51-62). Bad Heilbrunn: Klinkhardt.

Boger, M.-A. (2016). Zwischen Partikularisierung und Solidarisierung. Vortrag im Rahmen der Ringvorlesung „Behinderung ohne Behinderte?! Perspektiven der Disability Studies.“ Universität Hamburg. http://www.zedis-ev-hochschule-hh.de/files/boger_011116.pdf (Erstellt: 1. Nov. 2016). Zugegriffen: 9. Nov. 2020.

Bott, G. (1970). Erziehung zum Ungehorsam. Antiautoritäre Kinderläden. Frankfurt a.M.: Zweitausendeins.

Brunner, J. (2018). Professionalität in der Frühpädagogik. Perspektiven pädagogischer Fachkräfte im Kontext einer inklusiven Bildung. Wiesbaden: Springer VS.

Budde, J., \& Hummrich, M. (2015). Inklusion aus erziehungswissenschaftlicher Perspektive. In Deutsche Gesellschaft für Erziehungswissenschaft (Hrsg.), Inklusion - Perspektive, Herausforderung und Problematisierung aus Sicht der Erziehungswissenschaft. Mitteilungen der Deutschen Gesellschaft für Erziehungswissenschaft, (Bd. 26(51), S. 33-42). Opladen, Berlin, Toronto: Barbara Budrich.

DESTATIS (2020). Statistiken der Kinder- und Jugendhilfe. Kinder und tätige Personen in Tageseinrichtungen und in öffentlich geförderter Kindertagespflege am 01.03.2020. Wiesbaden: DESTATIS.

Freiling, T. (2003). Qualitätsentwicklung in selbstorganisierenden Systemen. Ein Beitrag zur Entwicklung der Organisationsqualität in Kinderbetreuungseinrichtungen freier Träger (Elterninitiativen). Kassel: university Press.

Gerstenberg, F., Riechers, K., Bruhn, A.-C., Folta-Schoofs, K., Haude, C., Miller, L., Bätge, C., \& Cloos, P. (2021). Within limits? Multidisziplinär forschen im Kontext der Frage nach einer Inklusiven Bildungsforschung der frühen Kindheit. In C. Bätge, P. Cloos, F. Gerstenberg \& K. Riechers (Hrsg.), Inklusive Bildungsforschung der frühen Kindheit. Empirische Perspektiven und multidisziplinäre Zugänge (S. 115-149). Weinheim: Beltz Juventa.

Gorden, R.L. (1975). Interviewing. Strategy, techniques and tactics. Homewood: Dorsey.

Hinz, A. (2010). Inklusion - historische Entwicklungslinien und internationale Kontexte. In A. Hinz, I. Körner \& U. Niehoff (Hrsg.), Von der Integration zur Inklusion. Grundlagen - Perspektiven Praxis (2. Aufl. S. 33-52). Marburg: Lebenshilfe Verlag.

Iseler, K. (2010). Kinderläden: Fallstudien zum Fortbestand sozialpädagogischer Organisationen. Münster: Waxmann.

Jansa, A. (2000). Die Pädagogik der Studentenbewegung in ihrer Auswirkung auf das Generationenverhältnis und den gesellschaftlichen Umgang mit Kindern. In Jahrbuch für Pädagogik 1999: Das Jahrhundert des Kindes (S. 223-246). Frankfurt a.M.: Peter Lang.

Katzenbach, D. (2015). De-Kategorisierung inklusive? Über Risiken und Nebenwirkungen des Verzichts auf Etikettierungen. In C. Huf \& I. Schnell (Hrsg.), Inklusive Bildung in Kita und Grundschule (S. 33-55). Stuttgart: Kohlhammer.

Kornmann, R. (1994). Von der prinzipiell nie falschen Legitimation negativer Ausleseentscheidungen zum Etikettierungs-Ressourcen-Dilemma oder: Gibt es überhaupt Perspektiven für eine förderungsorientierte Diagnostik? Behinderte in Familie, Schule und Gesellschaft, 17(H. 1), 51-59.

Kuckartz, U. (2016). Qualitative Inhaltsanalyse. Methoden, Praxis, Computerunterstützung (3. Aufl.). Weinheim: Beltz.

Link, J. (2013). Versuch über den Normalismus. Wie Normalität produziert wird (5. Aufl.). Göttingen: Springer VS. 
Meuser, M., \& Nagel, U. (2005a). ExpertInneninterviews - vielfach erprobt, wenig bedacht. Ein Beitrag zur qualitativen Methodendiskussion. In A. Bogner, B. Littig \& W. Menz (Hrsg.), Das Experteninterview. Theorie, Methode, Anwendung (2. Aufl. S. 71-93). Wiesbaden: Springer VS. (1991/2005).

Meuser, M., \& Nagel, U. (2005b). Vom Nutzen der Expertise ExpertInneninterviews in der Sozialberichterstattung. In A. Bogner, B. Littig \& W. Menz (Hrsg.), Das Experteninterview. Theorie, Methode, Anwendung (2. Aufl. S. 257-272). Wiesbaden: Springer VS.

Palm, R., \& Winkler, M. (2019). Der Kinderladen - eine Alternative im elementarpädagogischen Bereich (1996). In K. Bock, N. Göddertz, F. Heyden \& M. Mauritz (Hrsg.), Zugänge zur Kinderladenbewegung (S. 91-114). Wiesbaden: Springer VS. (1996/2019).

Przyborski, A., \& Wohlrab-Sahr, M. (2014). Qualitative Sozialforschung. Ein Arbeitsbuch. München: O1denbourg.

Reyer, J., \& Müller, U. (1992). Eltern-Kind-Gruppen. Eine neue familiale Lebensform? Freiburg im Breisgau: Lambertus.

Riechers, K., \& Baader, M. S. (2021). Inklusive Elterninitiativen (IKE). In C. Bätge, P. Cloos, F. Gerstenberg \& K. Riechers (Hrsg.), Inklusive Bildungsforschung der frühen Kindheit. Empirische Perspektiven und multidisziplinäre Zugänge (S. 35-38). Weinheim: Beltz Juventa.

Sander, A. (2004). Inklusive Pädagogik verwirklichen - Zur Begründung des Themas. In I. Schnell \& A. Sander (Hrsg.), Inklusive Pädagogik (S. 11-22). Bad Heilbrunn: Klinkhardt.

Schmid, P. (2008). Wie die antiautoritäre Erziehung für einige Jahre in städtische Kindertagesstätten gelangte. Das Frankfurter Modellprojekt Kita 3000, 1972-1978. In M.S. Baader (Hrsg.), ,Seid realistisch, verlangt das Unmögliche!" Wie 68 die Pädagogik bewegte (S. 16-35). Weinheim, Basel: Beltz.

Seifert, M. (1977). Zur Theorie der antiautoritären Kindergärten. In M. Seifert \& H. Nagel (Hrsg.), Nicht für die Schule leben. Freie Schule Frankfurt. Ein alternativer Schulversuch. Frankfurt a. M.: päd.extra.

Silvester, K. (2009). Der besseren Eltern?! Oder - Die Entdeckung der Kinderläden. ElternKind-Initiativen im zeitgeschichtlichen Vergleich 1967-2004. Eltern-Erwartungen und-Erfahrungen. Dissertation der Katholischen Universität Eichstätt

Wolff, R. (2019). Die ersten Kinderläden. Praxiserfahrungen und pädagogische Konzeptionen. In K. Bock, N. Göddertz, F. Heyden \& M. Mauritz (Hrsg.), Zugänge zur Kinderladenbewegung (S. 27-42). Wiesbaden: Springer VS. 